\title{
Evolution of fusion
}

The Lawrence Livermore National Laboratory in Livermore, California hosts the National Ignition Facility (NIF), currently the largest laboratory pursuing inertial confinement fusion (ICF). The idea is to use an intense $\mathrm{X}$-ray bath created when ultrafast laser pulses strike a gold or depleted uranium target to drive the implosion of a tiny fuel pellet containing hydrogen isotopes deuterium and tritium. In principle, the implosion could produce star-like pressures and temperatures, and energy from fusion reactions in excess of that required to produce the implosion.

This hasn't happened yet, but three years ago, the facility achieved a milestone experiments in which deuterium-tritium fusion reaction products, in colliding with other plasma particles, produced more fusion energy than did the initial work done on the fuel (O. A. Hurricane et al., Nat. Phys. $12,800-806 ; 2016)$. This isn't yet ignition, a propagating fusion reaction, which will require a pressure higher by a factor of two or so.

To get there, researchers at NIF plan to make incremental improvements reducing the influence of plasma instabilities or suppressing asymmetries in the implosion itself. The task amounts to a search for optimal performance within a complex space of design parameters. Any experiment requires a choice for the power, energy, frequency range and shape of the laser pulse, and for the gas filling the hohlraum - a hollow cylinder, at the centre of which resides the fuel pellet. One advance seems likely to come from changing the hohlraum from a cylindrical cavity to one shaped like a rugby ball (Y. Ping et al., Nat. Phys. 15, 138-141; 2019). Designers are also pursuing better choices for the structure and composition of the fuel pellet itself, where deuterium-tritium ice or gas can be mixed in innumerable layered patterns.

The current best designs have been found through laborious experimentation and human learning. But with so many design parameters - probably well over 100 - the space of possibilities remains only sparsely explored. Possibly, superior performance might be observed in regions of parameter space that to human intuition seem unpromising. That possibility is now motivating researchers to explore the ICF design space by using algorithms inspired by biological evolution.

Evolutionary algorithms aim to optimize within a space of designs not through any systematic search, but by harnessing the power of random exploration. The algorithms exploit the evolutionary recipe - selection, variation and iteration - by letting a population of possible designs reproduce, with the more successful having more offspring in the next generation. Mutations introduce true novelty into each generation, allowing new designs to be discovered. Selection ensures that designs improve over time. Such algorithms excel at exploring spaces where profitable combinations of features may not fit any obvious conceptual scheme.

Possibly, superior
performance
might be
observed in
regions of
parameter space
that to human
intuition seem
unpromising.

Performing an evolutionary search with real ICF experiments is currently out of the question, as it takes far too long to set up and run a single NIF 'shot'. So physicist Peter Hatfield of the University of Oxford and colleagues instead searched the design space in silico using simulations of the relevant physics (P. W. Hatfield et al., Phys. Plasmas (in the press); preprint at https://arxiv. org/abs/1905.08215; 2019). Fortunately, most of this physics - for radiation and hydrodynamics on dense, high-energy plasmas - is fairly well understood from experiments over several decades, and incorporated in a well-tested code, HYADES. In their initial work, Hatfield and colleagues used this code to evolve a population of designs.

To make the project manageable, they have so far only considered aspects of the ICF design downstream from the laser-hohlraum interaction, starting with the X-ray bath designed to compress the fuel pellet. Even here, the possible designs vary with some 60 parameters. With pellet designs of 5 layers, each layer can be deuterium gas or ice, or instead plastic, with differing densities, and each layer varying between $0.01 \mathrm{~mm}$ and $1.1 \mathrm{~mm}$. The X-ray drive has huge flexibility in design as a time sequence of 15 phases each represented by a peak temperature, time of peak and width.
The researchers started with 600 random initial designs and then ran the evolution for 80 generations, which required 48,000 HYADES simulations over 27 days.

Running this experiment three separate times - with different seeds - evolved three distinct final designs showing a roughly 20 -fold improvement over the 80 generations, all having a similar peak in the $\mathrm{X}$-ray drive and a mix of deuterium-tritium gas and ice in the interior of the capsule. It's not clear that any of these designs are in some sense global maxima within the considered parameter space, but that's not actually important. As the researchers point out, a global maximum within such a simplified 1D set-up isn't likely also to be a maximum for real experiments. Rather, the intention is to find plausible outlines of designs which can then be further refined.

Other researchers are also pursuing unconventional methods for experiment design in so-called direct-drive ICF, in which laser light illuminates the fuel target directly. Designs found through machine learning (V. Gopalaswamy et al., Nature 565, 581-586; 2019) or using methods for statistical inference (J. L. Peterson et al., Phys. Plasmas 24, 032702; 2017) have both demonstrated a significant boost in the yield achieved in real experiments. For either direct- or indirectdrive experiments, this kind of automated approach may help accelerate ICF progress by combining computational insight and qualitative human insight in an iterative way. Computation may point to designs that experienced physicists can rule as unphysical or impractical. But survivors may provide promising seeds to be explored with stateof-the-art 3D simulations, and finally - if still performing well - tested in real ICF experiments.

Nothing in this evolutionary approach, or in the other computational methods, is yet able to replace the most creative elements of the human designer, and make decisions on the most significant design features. But that may change with time as algorithms grow more powerful, and show signs of more general human intelligence. Even though the search for fusion energy success has been long and frustrating, progress could speed up greatly - and computation may be the key element that makes it happen.

\section{Mark Buchanan}

Published online: 1 July 2019

https://doi.org/10.1038/s41567-019-0574-9 